\title{
Clinical Applicability of Current Pharmacokinetic Models: Splanchnic Elimination of 5-Fluorouracil in Cancer Patients
}

\author{
Peter J. Robinson,, Ludvik Bass,, ${ }^{1,5}$ Susan M. Pond, ${ }^{2}$ Michael S. Roberts, ${ }^{3}$ \\ and John G. Wagner ${ }^{4}$
}

Received July 20, 1987-Final December 7, 1987

\begin{abstract}
What can be inferred from limited clinical data by using current models of hepatic elimination? We examined this question by analyzing previously published data on the steady-state uptake of the anticancer agent 5-fluorouracil (5-FU) in seven cancer patients in terms of the venous equilibration model, the undistributed and distributed forms of the sinusoidal perfusion model, and the convection-dispersion model. Because of appreciable extrasplanchnic removal of 5-FU, the value of the steady infusion rate was not used in our analysis. When the data from all patients were pooled by plotting the measured hepatic venous concentration against the measured hepatic arterial concentration, the high concentration data fell on a limiting straight line of slope 1 , indicating that at high dose rates elimination of 5-FU in both the liver and gastrointestinal tract was close to saturation. The intercept of this line gave a model-independent estimate of $V_{\max } / Q=$ $48.0 \pm 11.6(S D) \mu M$ for the pooled data set, where $V_{\max }$ is the maximum splanchnic elimination rate of 5-FU, and $Q$ is the hepatic blood flow. The low concentration data points fell on a limiting straight line through the origin, from which model-dependent values of the Michaelis constant were determined. The venous equilibration model gave $K_{\mathrm{m}}=9.4 \mu \mathrm{M}$, while the undistributed sinusoidal perfusion model gave $K_{\mathrm{m}}^{*}=26.5 \mu \mathrm{M}$. With these values of $K_{\mathrm{m}}$, both models fit the pooled data equally well. These methods were applied to analyses of the five individual data sets which contained sufficiently high concentration data points. The resulting mean values were $V_{\max } / Q=41.0 \pm 5.1(\mathrm{sem}) \mu M, K_{\mathrm{m}}=8.4 \pm 1.3 \mu M$ and $K_{\mathrm{m}}^{*}=23.2 \pm 3.2 \mu M$. However, the splanchnic region is a highly heterogeneous organ system, for which an undistributed analysis provides no more than an upper bound on the Michaelis constant $K_{\mathrm{m}}^{+}\left(K_{\mathrm{m}}^{+} \leq K_{\mathrm{m}}^{*}\right)$. A perfusion model distributed to represent total splanchnic elimination is developed in the Appendix. Using
\end{abstract}

\footnotetext{
We are grateful to the Australian Research Grants Scheme and the National Health and Medical Research Council for financial support, and to the Department of Science for the award of a National Research Fellowship to P.J.R.

'Department of Mathematics, University of Queensland, Brisbane, Australia.

${ }^{2}$ Department of Medicine, University of Queensland, Brisbane, Australia.

${ }^{3}$ School of Pharmacy, University of Otago, Dunedin, New Zealand.

${ }^{4}$ College of Pharmacy, Department of Pharmacology and Upjohn Center for Clinical Pharmacology, University of Michigan, Ann Arbor, Michigan.

${ }^{5}$ To whom correspondence should be addressed at Department of Mathematics, University of Queensland, St. Lucia, Queensland 4067, Australia.
} 
previous estimates of the degree of functional heterogeneity in the liver alone, this model yields $K_{\mathrm{m}}^{+}$values for individual patients which have a mean of $20.3 \pm 2.8 \mu \mathrm{M}$.

KEY WORDS: Michaelis-Menten kinetics; sinusoidal perfusion model; venous equilibration model; distributed model; convection-dispersion model; organ heterogeneity; bioavailability; pooling of data; kinetic parameter estimation.

\section{INTRODUCTION}

When substrates of hepatocellular enzymes are eliminated rapidly enough to display a first-pass effect and a hepatic clearance dependent on hepatic blood flow, any quantitative account of elimination and bioavailability of the substrate must somehow be related to hepatic physiology. Several quantitative liver models have been proposed for this purpose, and ingenious experiments with animal livers (both isolated and in situ) have been performed in order to discriminate between the models by precise statistical analyses. Such discrimination is not intended here. The present authors have put aside their differing views as to the relative merits of the models in order to examine another question: What is the impact of the several models on a particular set of clinical data from human subjects, typically more limited and less precise than data from animal preparations? Whatever may be the result of future discrimination between the models, interpretations of such data are the ultimate object of the modeling.

5-Fluorouracil (5-FU) has been used extensively for the treatment of solid tumors in man. It is eliminated by the liver, kidney, and other extrahepatic sites (1-3) but not by the lung (4). In particular, 5-FU is eliminated to a significant extent in the gastrointestinal (GI) tract (3). Chadwick and Rogers (2) found substantial concentrations of the metabolite $\alpha$-fluoro- $\beta$-ureidopropionic acid (FUPA) in the epithelium of the GI tract after intravenous injection of 5-FU in mice, indicating that enzymes for metabolic degradation of the drug are located in these epithelial cells.

In a recent study, Wagner et al. (5) investigated the hepatic elimination of 5-FU in man by making a series of three to six steady-state intravenous infusions of $2 \mathrm{hr}$ duration in seven cancer patients, while sampling the concentration of 5-FU in the hepatic artery $\left(C_{\mathrm{HA}}\right)$ and the hepatic vein $\left(C_{H V}\right)$. (Abbreviations, symbols, and Units used are shown in Table I.) Figure 1 presents a schematic diagram of the circulation showing the sites of the infusion and sampling catheters. The principal aim of the present paper is to devise methods to analyze this and other similar data sets in order to determine, for each individual patient, the appropriate kinetic parameters for uptake. We develop a method that is as far as possible independent of the assumptions of any particular mathematical model and unaffected by extrasplanchnic elimination. When a specific model of uptake 
Table I. Abbreviations, Symbols, and Units

\begin{tabular}{|c|c|}
\hline 5-FU & 5-Fluorouracil \\
\hline$C_{\mathrm{HA}}, C_{\mathrm{HV}}$ & Concentrations of $5-\mathrm{FU}$ in hepatic artery and hepatic vein $(\mu \mathrm{M})$ \\
\hline & Logarithmic mean of $C_{\mathrm{HA}}$ and $C_{\mathrm{HV}}[\mathrm{Eq} .(4)](\mu \mathrm{M})$ \\
\hline$Q$ & Total hepatic blood flow ( $\mathrm{L} \mathrm{min}^{-1}$, or $\mathrm{cm}^{3} \mathrm{sec}^{-1}$ ) \\
\hline$\lambda$ & Fraction of $Q$ passing through portal vein \\
\hline$V$ & Elimination rate of 5-FU in splanchnic region $\left(\mu\right.$ mole $\left.\min ^{-1}\right)$ \\
\hline$I$ & Total steady infusion of 5-FU $\left(\mu\right.$ mole $\left.\min ^{-1}\right)$ \\
\hline$V_{\max }$ & Maximum splanchnic elimination rate of 5.FU $\left(\mu\right.$ mole $\left.\min ^{-1}\right)$ \\
\hline$V_{\max }^{\mathrm{L}}, V_{\max }^{\mathrm{G}}$ & $\begin{array}{l}\text { Maximum elimination rate of 5-FU in liver and GI tract, respectively ( } \mu \text { mole } \\
\min ^{-1} \text { ) }\end{array}$ \\
\hline$K_{\mathrm{m}}$ & Michaelis constant $(\mu \mathrm{M})$ \\
\hline & $\begin{array}{l}\text { Superscripts referring to parameters estimated by the undistributed and dis- } \\
\text { tributed sinusoidal perfusion model, respectively }\end{array}$ \\
\hline$F_{\mathrm{i}}$ & Intrinsic bioavailability \\
\hline $\mathrm{Cl}_{\mathrm{i}}$ & Intrinsic clearance of total drug $\left(\mathrm{L} \mathrm{min}^{-1}\right)$ \\
\hline$\varepsilon, \varepsilon_{\mathrm{L}}, \varepsilon_{\mathrm{G}}$ & $\begin{array}{l}\text { Measures of the degree of functional heterogeneity in the total splanchnic } \\
\text { region, in the liver alone, and in the GI tract alone }\end{array}$ \\
\hline $\boldsymbol{R}$ & A positive remainder term [Eqs. (13) and (15)] \\
\hline$\rho$ & Hepatocellular enzyme density along sinusoids $\left(\mathrm{cm}^{-1}\right)$ \\
\hline A & Total cross-sectional area for flow through liver $\left(\mathrm{cm}^{2}\right)$ \\
\hline$L$ & Effective length of liver $(\mathrm{cm})$ \\
\hline$D$ & Dispersion coefficient for substrate passing through liver $\left(\mathrm{cm}^{2} \mathrm{sec}^{-1}\right)$ \\
\hline$D_{\mathrm{N}}$ & Dispersion number \\
\hline
\end{tabular}

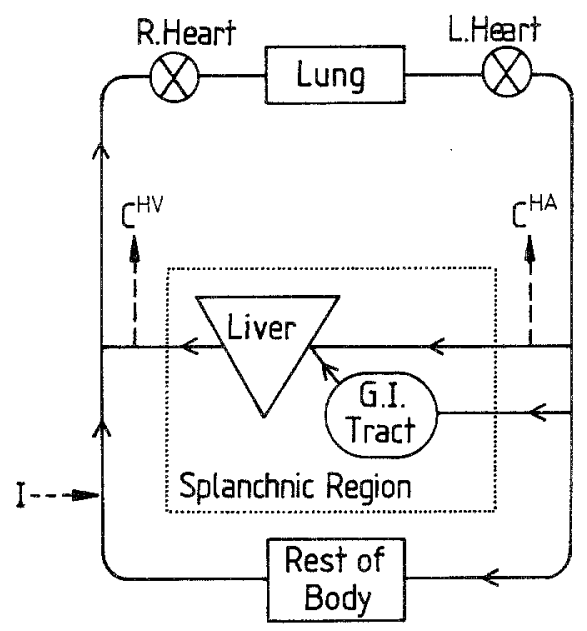

Fig. 1. Schematic diagram of the circulation, showing sites of sampling catheters in the hepatic artery $\left(C_{\mathrm{HA}}\right)$ and in the hepatic vein $\left(C_{\mathrm{HV}}\right)$, and the site of peripheral infusion I used in Wagner et al. (5). The dotted line encompasses the splanchnic region, consisting of the liver and the GI tract, connected by the portal vein. 
is necessary (as in the determination of the Michaelis constant $K_{\mathrm{m}}$ ), the data are analyzed according to a number of models currently in use, and their corresponding estimates of parameters are compared.

As elimination is steady, the concentration at entry to the GI tract is the same as $C_{\mathrm{HA}}$. Applying Fick's law in the steady state to the whole splanchnic region gives

$$
V=Q\left(C_{\mathrm{HA}}-C_{\mathrm{HV}}\right)
$$

where $V$ is the elimination rate of 5-FU in the splanchnic region consisting of the liver plus the GI tract (Fig. 1), and $Q$ is the total hepatic blood flow. Because no blood samples were taken from the portal vein between the GI tract and the liver, it is not possible to apportion the total splanchnic elimination rate $V$ between the liver and the GI tract. In our analysis of these data we therefore consider splanchnic elimination as a whole.

Because extrasplanchnic elimination of 5-FU in man is considerable (1), the splanchnic elimination rate $V$ in Eq. (1) is less than the total steady-state infusion rate, $I$. This is supported by the result of Wagner $e t$ $a l$. (5) that if $V$ is replaced by $I$ in Eq. (1), then each set of data $I, C_{\mathrm{HA}}$, $C_{\mathrm{HV}}$ gives a calculated value of $Q$ much greater than hepatic blood flow (as high as the cardiac output). We therefore calculate $V$ directly from Eq. (1) using measured steady-state values for $C_{\mathrm{HA}}$ and $C_{\mathrm{HV}}$, and either measured or estimated values for the hepatic blood flow $Q$.

In order to estimate kinetic parameters for the splanchnic uptake of 5-FU in man, a model for such elimination in the liver and the GI tract is required. A number of models currently in use describe the saturable uptake of substances by the intact liver. These include the undistributed sinusoidal perfusion model (6); the venous equilibration model (7-9); the distributed sinusoidal perfusion model (10), and the convection-dispersion model (11-13). Although all these models assume that Michaelis-Menten kinetics hold for the interaction of substrate and enzyme, they differ in their assumptions about the manner of presentation of substrate to enzyme in the physiological setting. The venous equilibration model assumes that all hepatocytes are presented with the concentration of substrate observed in the emergent venous blood. This model was developed for the liver alone and is inappropriate for elimination by the entire splanchnic region. (Are the enzymes in the GI tract to operate at a substrate concentration equal to that in the hepatic vein?) The undistributed sinusoidal perfusion model $(6,14)$ is only slightly more realistic in this case. This model assumes that the entire organ consists of functionally identical passageways (sinusoids), lined with enzymes, through which the substrate passes and is progressively depleted, and that all sinusoids output the same substrate concentration into the hepatic vein. However, considering the typical division of the 
hepatic blood flow between the hepatic artery and the portal vein, approximately two-thirds of the substrate passes through both the GI tract and the liver, while the rest passes through the liver alone (Fig. 1). Such heterogeneity in the handling of substrate must be taken into account. This can be accomplished in two related ways. First, the GI tract and the liver can be modeled separately and then put together into a composite model, with the hepatic arterial blood mixing with the portal venous blood from the GI tract to form the input for the liver. A model of this kind is described in the Appendix. More generally, the splanchnic region as a whole can be described in terms of a single distributed (heterogeneous) model. Two such models have been developed and are currently in use to describe hepatic elimination of substrate.

The distributed sinusoidal perfusion model (10) allows sinusoidal blood flows and enzyme contents to vary among sinusoids, with substrate output from each sinusoid mixing in the hepatic vein to form the measured output concentration $C_{\mathrm{Hv}}$. The output concentration from such a heterogeneous liver is always greater than that from an equivalent undistributed liver with the same input concentration, and with the same total enzyme content and blood flow, divided equally among all sinusoids (10). More recently, the concept of independent, noninteracting sinusoids has been modified to allow for the effects of intrahepatic mixing sites (13). The convectiondispersion model of Roberts and Rowlands $(11,12)$ allows heterogeneity of substrate transits through the liver by a different process: it abandons entirely the concept of independent sinusoids in favor of combining unidirectional convection with a generalized term to account for dispersion of substrate passing through the liver.

All four models of hepatic elimination have markedly different mathematical forms and therefore may make quite different predictions as to the outcome of experiments, particularly if these experiments involve changes in hepatic blood flow. Although both the undistributed sinusoidal perfusion model and the venous equilibration model have received some experimental support, both these models have been refuted (11-20). Despite such experimental refutations, and the fact that these two models are inappropriate to describe total splanchnic elimination in detail, they have the advantage of not requiring an additional parameter to specify the heterogeneity in flow and enzyme activity. It is therefore useful to analyze the data of Wagner $e t$ al. (5) in terms of both these models (as well as the more appropriate distributed models). As all models share certain limiting features, some kinetic parameters, but not others, depend on the choice of model. In particular, the maximum elimination capacity of the liver (plus GI tract) is model-independent, whereas the Michaelis constant $K_{\mathrm{m}}$ is highly modeldependent. The value of $K_{\mathrm{m}}$ obtained by fitting the data to the undistributed 
perfusion model has the additional use of providing an upper bound to any $K_{\mathrm{m}}$ obtained by fitting the same data to the distributed perfusion model.

\section{MODELS OF HEPATIC ELIMINATION}

The venous equilibration model (7-9) asserts that all hepatocytes are exposed to substrate concentrations equal to the hepatic venous concentration $C_{\mathrm{HV}}$. For Michaelis-Menten kinetics, the elimination capacity of the liver is then given by

$$
V=V_{\max }\left[\frac{C_{\mathrm{HV}}}{C_{\mathrm{HV}}+K_{\mathrm{m}}}\right]
$$

where $V_{\max }$ is the maximum hepatic elimination rate, and $K_{\mathrm{m}}$ is the Michaelis constant.

The undistributed perfusion model (6), on the other hand, takes into account the depletion of substrate along the hepatic blood flow so that hepatocytes further downstream are exposed to progressively lower substrate concentrations. Those at the arterial end operate at the common input (arterial) substrate concentration $C_{\mathrm{HA}}$, while those at the venous end operate at the concentration $C_{\mathrm{HV}}$. The total elimination rate is then given by (6)

$$
V=V_{\max }^{*}\left[\frac{\hat{C}}{\hat{C}+K_{\mathrm{m}}^{*}}\right]
$$

where

$$
\hat{C}=\left(C_{\mathrm{HA}}-C_{\mathrm{HV}}\right) / \ln \left(C_{\mathrm{HA}} / C_{\mathrm{HV}}\right)
$$

is the logarithmic mean of $C_{\mathrm{HA}}$ and $C_{\mathrm{HV}}: C_{\mathrm{HA}}>\hat{C}>C_{\mathrm{HV}}$. When applied to the same data set of $C_{\mathrm{HA}}, C_{\mathrm{HV}}$ values, these two models generally yield different values of the kinetic parameters $V_{\max }$ and $K_{\mathrm{m}}$. We thus follow the convention of Wagner et al. (5) and denote values fitted by the sinusoidal perfusion model with asterisks [Eq. (3)] while omitting them from values fitted by the venous equilibration model [Eq. (2)]. The measurable quantities $C_{\mathrm{HA}}, C_{\mathrm{HV}}$, and $V$ are common to all models.

\section{Limiting Straight Lines}

For high venous substrate concentrations, $C_{\mathrm{HV}} \gg K_{\mathrm{m}}$ in Eq. (2); $\hat{C} \gg K_{\mathrm{m}}^{*}$ in Eq. (3), we have, using also Eq. (1)

$$
V=Q\left(C_{\mathrm{HA}}-C_{\mathrm{HV}}\right)=V_{\max }=V_{\max }^{*}
$$

Thus, at sufficiently high substrate concentrations, the hepatic elimination rate approaches a maximum value that is common to all models that assume 
Michaelis-Menten kinetics locally: all the enzyme molecules are saturated. In a plot of $C_{\mathrm{HV}}$ against $C_{\mathrm{HA}}$, we thus expect from Eq. (5) a limiting straight line at high concentrations, with a slope of 1 and an intercept with the abscissa at $C_{\mathrm{HA}}=V_{\max } / Q=V_{\max }^{*} / Q$. In what follows, we assume that the maximum elimination capacity of the liver can be determined in such a model-independent manner from its elimination rate measured at concentrations close to those for enzyme saturation. We therefore drop the asterisk on $V_{\max }$ determined from the sinusoidal perfusion model. Indeed, at high substrate concentrations, Eq. (5) applies to all models, including the distributed perfusion and dispersion models: If all enzyme molecules are saturated and working at maximum capacity, the maximum elimination rate of substrate for the organ as a whole is simply the sum total of enzymatic maximum elimination rates, regardless of how those enzymes are distributed among the sinusoids, or the manner of extent of dispersion of substrate within the liver. Thus Eq. (5) represents the limiting straight line for all models at high substrate concentration, yielding a model-independent estimate of $V_{\max } / Q$.

At the opposite extreme of low substrate concentrations, another limiting straight line is found in a plot of $C_{\mathrm{HV}}$ vs. $C_{\mathrm{HA}}$. For $C_{\mathrm{HV}} \ll K_{\mathrm{m}}$ in Eq. (2) we have, using Eq. (1) and rearranging

$$
\frac{C_{\mathrm{HV}}}{C_{\mathrm{HA}}}=\frac{1}{1+\frac{V_{\max }}{Q K_{\mathrm{m}}}}
$$

On the other hand, for $\hat{C} \ll K_{\mathrm{m}}^{*}$ in Eq. (3) we have, again using Eq. (1)

$$
V=Q\left(C_{\mathrm{HA}}-C_{\mathrm{HV}}\right)=V_{\max } \frac{\hat{C}}{K_{\mathrm{m}}^{*}}
$$

Using the definition of $\hat{C}$ [Eq. (4)] and canceling $\left(C_{\mathrm{HA}}-C_{\mathrm{HV}}\right)$ from both sides, we obtain, on rearrangement

$$
\frac{C_{\mathrm{HV}}}{C_{\mathrm{HA}}}=e^{-V_{\text {max }} / Q K_{\mathrm{m}}^{*}}
$$

Equation (8) is derived in a more general way in Bass et al. (6), where it is shown that Eq. (8) holds provided either that $V / Q K_{\mathrm{m}}^{*} 1$ or that $V / V_{\max } \ll 1$.

Both Eqs. (6) and (8) describe a direct proportionality between $C_{\mathrm{HV}}$ and $C_{\mathrm{HA}}$ at low substrate concentrations

$$
C_{H V}=F_{\mathrm{i}} C_{\mathrm{HA}}
$$

where the constant of proportionality $F_{\mathrm{i}}$ is the intrinsic bioavailability, defined as the low concentration (first-order) limit of $\left(C_{\mathrm{HV}} / C_{\mathrm{HA}}\right)$. The 
interpretation of $F_{\mathrm{i}}$ in terms of the kinetic parameters, particularly $K_{\mathrm{m}}$, is highly model dependent. For the venous equilibration model we have, from Eqs. (6) and (9)

$$
F_{\mathrm{i}}=\frac{1}{1+\frac{V_{\max }}{Q K_{\mathrm{m}}}}=\frac{1}{1+\frac{C l_{\mathrm{i}}}{Q}}
$$

where $C l_{\mathrm{i}}=V_{\max } / K_{\mathrm{m}}$ is the intrinsic hepatic clearance of total drug according to the venous equilibration model. For the sinusoidal perfusion model, on the other hand, Eqs. (8) and (9) give

$$
F_{\mathrm{i}}=e^{-V_{\max } / Q K_{\mathrm{m}}^{*}}=e^{-C l_{\mathrm{w}}^{*} / Q}
$$

where $C l_{i}^{*}=V_{\max } / K_{\mathrm{m}}^{*}$ is the intrinsic clearance according to the undistributed perfusion model. The intrinsic bioavailability $F_{\mathrm{i}}$ is determined directly from the data and is model-independent. It is the interpretation of $F_{i}$ in terms of the kinetic parameters that is highly model-dependent, with Eqs. (10) and (11) giving interpretations of $F_{\mathrm{i}}$ according to the venous equilibration and sinusoidal perfusion models respectively. We note that Eq. (11) can be written, on expanding the exponential, as

$$
F_{\mathrm{i}}=\frac{1}{e^{C l_{*}^{*} / Q}}=\frac{1}{1+C l_{\mathrm{i}}^{*} / Q+\frac{1}{2}\left(C l_{\mathrm{i}}^{*} / Q\right)^{2}+\cdots}
$$

It is important to notice that if the development of the denominator is broken off after the linear term and the resulting expression is combined with Fick's law [Eq. (1)], one gets the same relation between $F_{\mathrm{i}}$ and $Q$ as Eq. (10) in the venous equilibration model: hence $C l_{\mathrm{i}}^{*} \approx C l_{\mathrm{i}}\left(K_{\mathrm{m}}^{*} \approx K_{\mathrm{m}}\right)$ from any given data. The two models differ therefore inasmuch as the terms so discarded are quantitatively important. It follows that the smaller $C \mathrm{l}_{\mathrm{i}}^{*} / Q$, the less the predictions of the two models differ (11). The distributed sinusoidal perfusion model and the convection-dispersion model also predict limiting straight lines in a plot of $C_{\mathrm{HV}}$ against $C_{\mathrm{HA}}$ at both high and low substrate concentrations.

In the first-order limit of low substrate concentrations the distributed sinusoidal perfusion model predicts the following relation between $C_{\mathrm{HA}}$ and $C_{\mathrm{HV}}(15)$

$$
F_{\mathrm{i}}=\frac{C_{\mathrm{HV}}}{C_{\mathrm{HA}}}=\exp \left\{-\frac{V_{\max }}{Q K_{\mathrm{m}}^{+}}+\frac{1}{2} \varepsilon^{2}\left[\frac{V_{\max }}{Q K_{\mathrm{m}}^{+}}\right]^{2}-R\right\}
$$

where $\varepsilon$ is the coefficient of variation of the (flow-weighted) distribution of maximum sinusoidal arterial-venous concentration differences, and is a measure of the degree of functional heterogeneity of the liver. For a liver 
made up of a number of sinusoidal groups in parallel ("notional suborgans"), each consisting of identical sinusoids and having its own $V_{\max }^{(p)}$ and $Q^{(\mathrm{p})}$, where $\Sigma_{\mathrm{p}} V_{\max }^{(\mathrm{p})}=V_{\max }$ and $\Sigma_{\mathrm{p}} Q^{(\mathrm{p})}=Q$, it has been shown that $\varepsilon^{2}$ is given by (19)

$$
\varepsilon^{2}=\sum_{\mathrm{p}} \frac{\left.V_{\max }^{(\mathrm{p})} / V_{\max }\right)^{2}}{Q^{(\mathrm{p})} / Q}-1
$$

The superscript "p" is chosen to denote parallel elements. $R$ is a positive remainder term for which there are precise upper and lower bounds, provided that $\varepsilon$ is small enough for the quantity $v_{0}=1 / \varepsilon-\varepsilon V_{\max } / Q K_{\mathrm{m}}^{*}$ to be positive (15):

$-\ln \left\{1-\frac{e^{-v_{0}^{2} / 2}}{\sqrt{ } 2 \pi\left[\frac{v_{0}}{2}+\frac{v_{0}^{2}}{4}+1\right]}\right\}<R \leq-\ln \left\{1-\frac{e^{-v_{0}^{2} / 2}}{\sqrt{ } 2 \pi\left[\frac{v_{0}}{2}+\frac{v_{0}^{2}}{4}+\frac{2}{\pi}\right]}\right\}$

It is apparent that as $\varepsilon^{2}$ decreases, $R$ falls off much faster than $\varepsilon^{2}$.

The symbol $(+)$ is placed on the $K_{\mathrm{m}}$ in Eq. (13) to indicate that the $K_{\mathrm{m}}$ fitted to data according to the distributed model in general differs from $K_{\mathrm{m}}^{*}$ fitted by the undistributed model and $K_{\mathrm{m}}$ fitted by the venous equilibration model. For experimentally determined values of $F_{\mathrm{i}}, V_{\max }$, and $Q$, it follows from a comparison of Eqs. (8) [or (11)] and (13) that $K_{\mathrm{m}}^{+} \leq K_{\mathrm{m}}^{*}$ [provided that $R \ll \frac{1}{2} \varepsilon^{2}\left(V_{\max } / Q K_{\mathrm{m}}^{+}\right)^{2}$ ]. Thus the distributed sinusoidal perfusion model fits the same low concentration-limiting straight line in a plot of $C_{\mathrm{HV}}$ against $C_{\mathrm{HA}}$ by assigning a smaller value to $K_{\mathrm{m}}^{+}$than $K_{\mathrm{m}}^{*}$. In general, the larger the degree of heterogeneity (represented by the parameter $\varepsilon$ ), the more $K_{\mathrm{m}}^{+}$falls below $K_{\mathrm{m}}^{*}$. Thus $K_{\mathrm{m}}^{*}$ provides a useful upper bound on $K_{\mathrm{m}}^{+}$ in cases where the magnitude of $\varepsilon$ cannot be estimated directly.

The recently introduced convection-dispersion model of Roberts and Rowland $(11,12)$ adds a generalized dispersion term (acting in the direction of flow) to the convection of substrate through the liver. In the limit of first-order uptake of substrate, there is a mathematical correspondence between the dispersion coefficient $D$ so introduced (mathematically analogous to, though much greater than, the molecular diffusion coefficient of the substrate) and the coefficient of variation $\varepsilon$ of the distributed model $(11,13)$

$$
\varepsilon^{2}=2 \frac{\overline{\rho^{2}}}{\bar{\rho}^{2}} \frac{A D}{Q L}
$$

where $A$ is the total cross-sectional area for flow through the liver, and $\rho$ is the hepatocellular enzyme density along the effective length $L$ of the liver; $\bar{\rho}$ and $\overline{\rho^{2}}$ are the mean and mean-square values of $\rho$, and the ratio $\rho^{2} / \bar{\rho}^{2} \geq 1$ expresses the effect of nonuniformity of enzyme distributions in 
the direction of flow (13). The dimensionless quantity $A D / Q L$, called the dispersion number $D_{\mathrm{N}}$, must be $\ll 1$ for the relation (16) to hold. In the first-order limit, therefore, the convection-dispersion model also predicts a straight line through the origin in a plot of $C_{\mathrm{HV}}$ against $C_{\mathrm{HA}}$, with the slope $F_{\text {i }}$ given by Eq. (13) (with $R=0$ ), and $\varepsilon$ of the distributed sinusoidal perfusion model identified with the corresponding quantity in the convection-dispersion model, given by Eq. (16).

\section{ANALYSIS OF DATA}

\section{Pooled Data}

We now turn in detail to the data of Wagner et al. (5), in which the steady-state hepatic arterial and hepatic venous concentrations of 5-FU were measured during steady infusions of 5-FU into a peripheral vein (Fig. 1). From three to six infusions were performed on each of seven patients. Figure 2 shows these data in the form of a plot of $C_{\mathrm{HV}}$ against $C_{\mathrm{HA}}$. Such straightforward pooling of data of this form is successful because

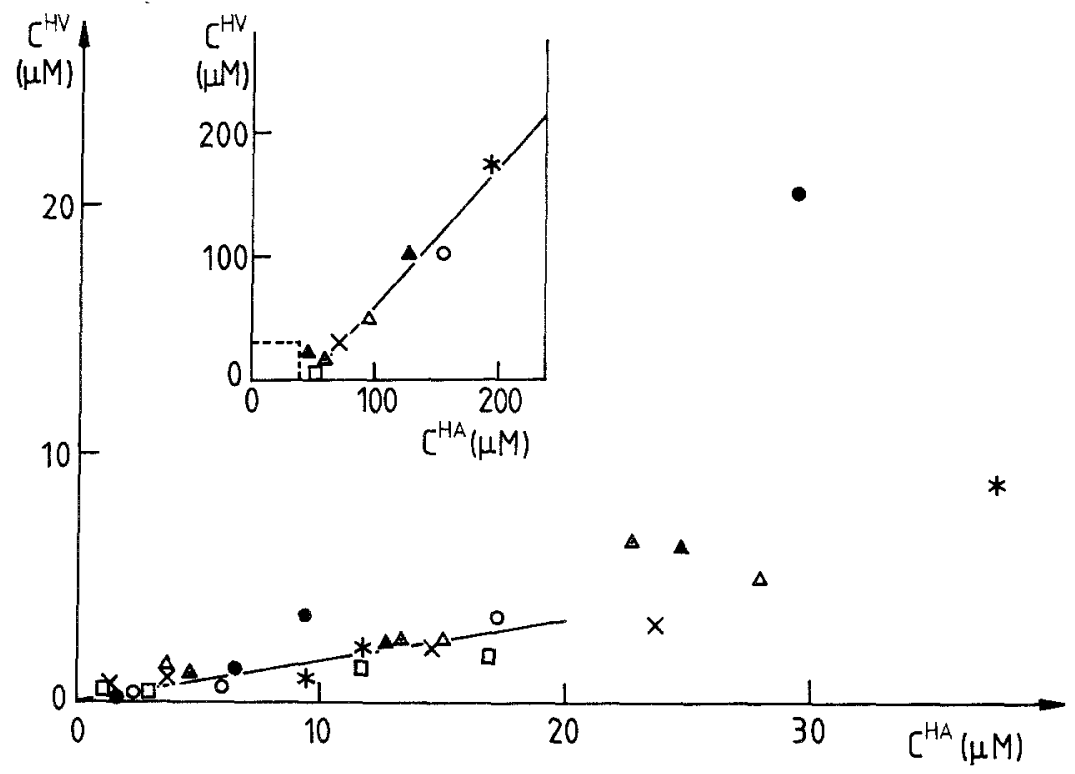

Fig. 2. Plot of steady-state hepatic venous concentration $C_{H V}$ vs. hepatic arterial concentration $C_{\mathrm{HA}}$ in seven cancer patients. Data are from Wagner et al. (5). The straight line is the linear regression through 20 data points with $C_{\mathrm{HA}}<20 \mu \mathrm{M}$, constrained to pass though the origin. Inset shows high concentration data points $\left(C_{\mathrm{HA}}>40 \mu \mathrm{M}\right)$. The straight line represents the linear regression through the five highest concentration points, which intercepts the abscissa at $V_{\text {max }} / Q=48.0 \pm 11.6$ (SD) $\mu \mathrm{M}$ and has a slope of $1.09 \pm 0.14$ (SD). 
at both high and low substrate concentrations the limiting straight lines are determined in all models by the ratio $V_{\max } / Q$ (as well as by $K_{\mathrm{m}}$ ) and not by $V_{\max }$ and $Q$ separately: see Eqs. (5), (6), (8), and (13). Thus biological variations in liver mass, to which both $V_{\max }$ and $Q$ would be expected to be roughly proportional, cancel at both these limits. Such pooling would not in general be as successful at intermediate substrate concentrations where $V_{\max }$ and $Q$ exert their influences separately.

The limiting straight lines described in the section "Models of Hepatic Elimination" are recognizable in Fig. 2. Independent estimates of $K_{\mathrm{m}}$ for uptake of 5-fluorouracil by isolated rat hepatocytes (21) are between 12 and $37 \mu \mathrm{M}$. Consistent with these values, examination of Fig. 2 . indicates that data points with $C_{\mathrm{HA}} \geq 70 \mu \mathrm{M}$ may be considered as determining the high concentration-limiting straight line, Eq. (5). A linear regression through these five highest concentration points has a slope of $1.09 \pm 0.14$ (SD) and an intercept $V_{\text {max }} / Q=48.0 \pm 11.6$ (SD) $\mu \mathrm{M}$ (see Fig. 2, inset). The slope is not significantly different from unity, which implies that at such high concentrations of 5-FU, elimination by both the liver and the GI tract is saturated. This precludes the possibility of significant elimination of 5-FU in the GI tract by a nonsaturable process such as passive loss into the intestinal lumen. This is consistent with the results of Chadwick and Rogers (2), who showed that after intravenous injections of 5-FU, metabolites of 5-FU found in the gastrointestinal endothelium and in the liver were similar. This indicates that similar enzymatic elimination processes are taking place in the GI tract as in the liver.

The limiting straight line through the origin is apparent for data points with $C_{\mathrm{HA}} \leqslant 20 \mu \mathrm{M}, C_{\mathrm{HV}} \leqslant 5 \mu \mathrm{M}(\hat{C} \leqslant 10 \mu \mathrm{M})$. This range is consistent with $K_{\mathrm{m}}$ values measured for isolated rat hepatocytes (21). We thus use the 20 lowest concentration data points to estimate $F_{\mathrm{i}}$ using Eq. (9) by means of linear regression constrained to pass through the origin (Fig. 1), giving $F_{\mathrm{i}}=0.163$. This intrinsic bioavailability, $F_{\mathrm{i}}$, given directly by observables, is model-independent. However, its interpretation in terms of the kinetic parameters $K_{\mathrm{m}}, V_{\max }$, and $Q$ is model-dependent. For the venous equilibration model, $F_{\mathrm{i}}$ is given by Eq. (10). Using $V_{\max } / Q=48.0 \mu \mathrm{M}$ gives $K_{\mathrm{m}}=$ $9.4 \mu \mathrm{M}$, while for the same value of $V_{\max } / Q$ the sinusoidal perfusion model [Eq. (11)] gives $K_{\mathrm{m}}^{*}=26.5 \mu \mathrm{M}$. Without an estimate of the degree of functional heterogeneity of the total splanchnic region consisting of liver plus GI tract, characterized by the parameter $\varepsilon^{2}$, we cannot make an outright estimate of $K_{\mathrm{m}}^{+}$, the value of the Michaelis constant predicted by Eq. (13) of the distributed sinusoidal perfusion model. However, as noted in "Models of Hepatic Elimination," $K_{\mathrm{m}}^{+} \leq K_{\mathrm{m}}^{*}$, so that $K_{\mathrm{m}}^{+} \leq 26.5 \mu \mathrm{M}$ from the pooled data of Wagner et al. (5). Closer estimates of $K_{\mathrm{m}}^{+}$are given in the Appendix and Conclusions. 
We note that in the context of the venous equilibration model, the 20 data points used to determine $F_{\mathrm{i}}$ have $C_{\mathrm{HV}}$ values less than about $40 \%$ of our estimated value of $K_{\mathrm{m}}(9.4 \mu \mathrm{M})$, thus validating their use in the determination of $F_{\mathrm{i}}$. The five highest concentration data points have $C_{\mathrm{HV}}$ values more than three times the estimated value of $K_{\mathrm{m}}$, justifying their inclusion in the determination of $V_{\max } / Q$. Similarly for the sinusoidal perfusion model, the highest value of $\hat{C}$ of the 20 data points used in the determination of $F_{\mathrm{i}}$ is less than one-third of $K_{\mathrm{m}}^{*}(26.5 \mu \mathrm{M})$, whereas the 5 highest concentration data points have $\hat{C}$ values of more than 1.8 times $K_{\mathrm{m}}^{*}$.

\section{Analysis of Individual Patients}

Using the information gained from our analysis of the pooled data from all seven cancer patients studied by Wagner et al. (5) (by their Method 1), we devised methods for analyzing the fewer data from each individual patient. In particular, only four of the seven individual data sets included a steady-state hepatic arterial concentration $C_{\mathrm{HA}} \geq 70 \mu \mathrm{M}$. (This included Patient \#4, who was studied on two separate occasions). From our study of the pooled data (Fig. 2), we saw that the slope of the straight line through the five highest concentration data points was not statistically significantly different from 1, indicating saturation of both the liver and the GI tract at these concentrations. We therefore calculated $V_{\max } / Q$ for each of these patients from their single highest concentration data point using Eq. (5). (This is equivalent, in a plot of $C_{\mathrm{HV}}$ against $C_{\mathrm{HA}}$, to drawing a straight line with slope 1 through the relevant point, with the intercept of this straight line with the abscissa giving $V_{\max } / Q$.) Values of $V_{\max } / Q$ calculated in this way are given in Table II, and have a mean value of $41.0 \pm 5.1$ (SEM) $\mu \mathrm{M}$, compared with $48.0 \mu \mathrm{M}$ for the pooled data.

The intrinsic bioavailability $F_{\mathrm{i}}$ can be determined for each individual patient in the same way as for the pooled data set, by forming a linear regression through the lowest concentration data points $C_{\mathrm{HV}}, C_{\mathrm{HA}}$, constrained to pass through the origin. Restricting ourselves to values of $C_{\mathrm{HA}} \leq 20 \mu \mathrm{M}$ as before, we find that most patients have three or more data points in this range (Table II). Values of $F_{\mathrm{i}}$ calculated for individual patients are given in Table II. The mean of the individual $F_{\mathrm{i}}$ determinations is $0.179 \pm 0.023(\mathrm{SEM})$, which compares with $F_{\mathrm{i}}=0.163$ obtained from the pooled data.

As with the pooled data analysis, in order to determine $K_{\mathrm{m}}$ values from the individual $F_{\mathrm{i}}$ values, a particular liver model must be chosen. For the venous equilibration model, Eq. (10) was used with individual values of $V_{\text {max }} / Q$ (where available) to determine the $K_{\mathrm{m}}$ values given in Table II. These have a mean of $8.4 \pm 1.3(\mathrm{SEM}) \mu \mathrm{M}$. For the sinusoidal perfusion 


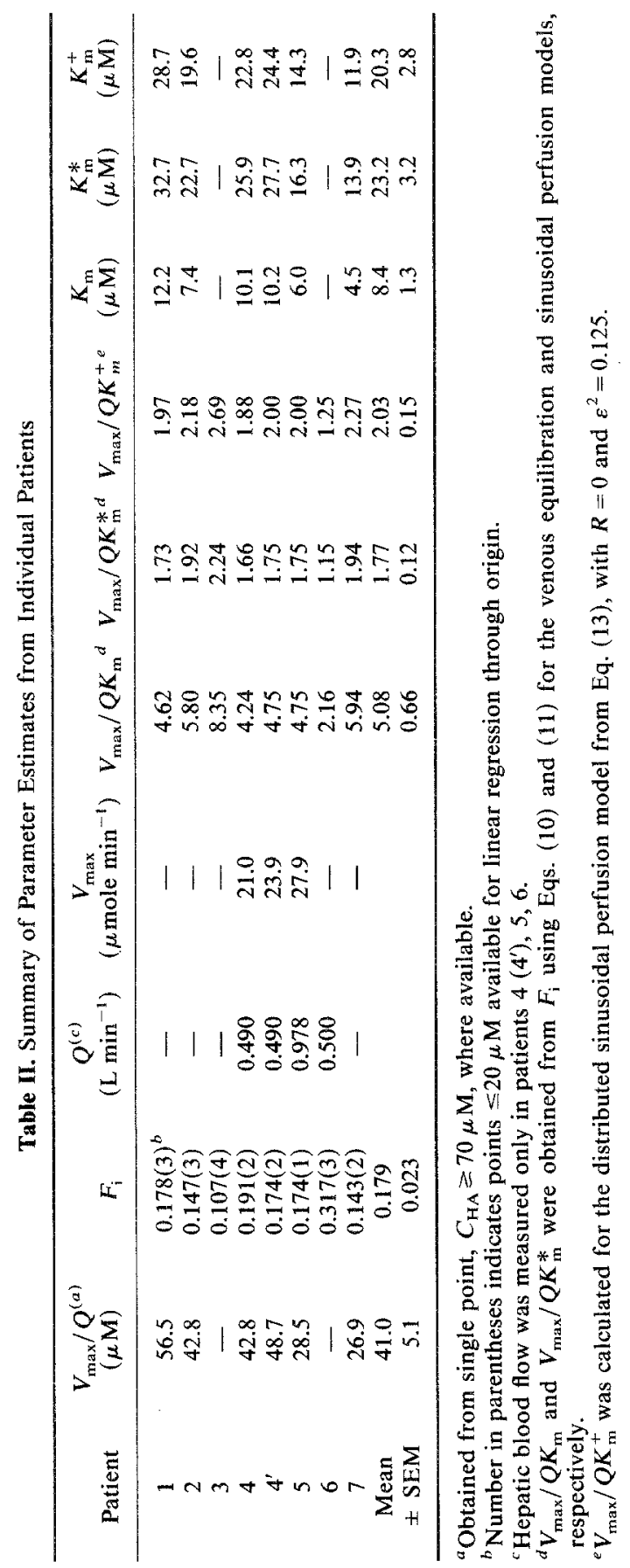


model, we used Eq. (11), again with individual $V_{\max } / Q$ values to obtain $K_{\mathrm{m}}^{*}$ values with a mean of $23.2 \pm 3.2$ (SEM) $\mu \mathrm{M}$. These compare favorably with values obtained from the pooled data of $9.4 \mu \mathrm{M}$ and $26.5 \mu \mathrm{M}$ for the venous equilibration and sinusoidal perfusion models respectively.

Values for the hepatic blood flow $Q$ were measured directly in three patients (Table II), allowing values of the maximum elimination rate $V_{\max }$ to be determined from the $V_{\max } / Q$ ratios. These values ranged from 21.0 to $27.9 \mu$ mole $\min ^{-1}$ (Table II).

\section{CONCLUSIONS}

When $C_{\mathrm{HA}}$ and $C_{\mathrm{HV}}$ are measured across the Michaelis-Menten range of concentrations (above and below $K_{\mathrm{m}}$ ), two model-independent quantities may be determined, one from the limit of high (saturating) substrate concentrations, and the other from the first-order limit at low substrate concentrations, at which $C_{\mathrm{HV}}$ is proportional to $C_{\mathrm{HA}}$. At high concentrations, a plot of $C_{\mathrm{HV}}$ against $C_{\mathrm{HA}}$ for all seven patients studied by Method 1 of Wagner et al. (5) tends to a limiting straight line with a slope not significantly different from 1 (Fig. 2, inset), indicating that elimination in both the liver and GI tract is saturated. This result precludes significant first-order elimination in the GI tract (for example, by diffusion of 5-FU into the lumen). The intercept of the limiting straight line with the abscissa gives an estimate of $V_{\max } / Q$ for the pooled data, where $V_{\max }=V_{\max }^{\mathrm{L}}+V_{\max }^{\mathrm{G}}$ is the sum of the maximum elimination rates of the liver and the GI tract, of $48.0 \pm 11.6$ (SD) $\mu \mathrm{M}$ (see the section "Analysis of Data" and Fig. 2, inset). The contributions of the liver and the GI tract to this total could not be separated because the 5-FU concentration in the portal vein was not sampled. Estimates of $V_{\text {max }} / Q$ for individual patients are given in Table II. In those cases in which a value for the hepatic blood flow $Q$ is also available, an estimate of $V_{\max }$ is also given in Table II. Estimates of $V_{\max } / Q$ (or $V_{\max }$ ) calculated in this way are common to all the models of elimination discussed in this paper, and are thus considered model-independent in the present context.

At the other extreme of low substrate concentrations $\left(C_{\mathrm{HA}} \ll K_{\mathrm{m}}\right)$, all models predict direct proportionality between $C_{\mathrm{HV}}$ and $C_{\mathrm{HA}}$ : a plot of $C_{\mathrm{HV}}$ against $C_{\mathrm{HA}}$ tends towards a limiting straight line through the origin as low substrate concentrations are approached. Such a limiting straight line is clearly observed in Fig. 2 in the pooled 5-FU uptake data of Wagner et al. (5). However, the models differ markedly in their interpretation of the slope of this straight line-the intrinsic bioavailability $F_{\mathrm{i}}$-in terms of the parameters $V_{\max }, Q$, and $K_{\mathrm{m}}$. Thus, for the pooled data, the venous equilibration model gives an estimate of $K_{\mathrm{m}}$ of $9.4 \mu \mathrm{M}$, whereas the undistributed sinusoidal perfusion model gives $K_{\mathrm{m}}^{*}=26.5 \mu \mathrm{M}$. With these values of $K_{\mathrm{m}}$, 
both models fit the data equally well (for the totality of pooled data points, the sum of squares of differences between predicted and measured values of $C_{\mathrm{HV}}$ are 1338 and $1505 \mu \mathrm{M}^{2}$ for the venous equilibration and sinusoidal perfusion models, respectively). It is interesting (though not essential in our analysis) that both these values of $K_{\mathrm{m}}$ are close to or within the range of between $12 \mu \mathrm{M}$ and $37 \mu \mathrm{M}$ reported for isolated rat hepatocytes by Mentre et al. (21).

As noted in the Introduction, the anatomy and the arrangement of blood flow through the total splanchnic region do not lend themselves to a direct application of either the venous equilibration or the undistributed sinusoidal perfusion model, even if we disregard the refutations of these models in isolated livers $(11-13,15-18,20)$. In addition, analyses of the uptake of various substrates by the intact liver have shown that the liver alone has a significant degree of functional heterogeneity characterized by the parameter $\varepsilon^{2}$ (see, for example, refs. 10, 15, 19, 20). In the Appendix we therefore outline a composite model of the splanchnic region in which a minimum degree of heterogeneity is taken into account. The liver and GI tract are modeled according to the distributed and undistributed forms of the sinusoidal perfusion model, respectively. A composite eliminating organ is then modeled by assuming that blood from the hepatic artery mixes with blood from the portal vein before entry into the liver. The heterogeneity of this model is characterized by the parameter $\varepsilon$ given by Eq. (A3). Gustavsson et al. (3) have estimated that in mice, some $15 \%$ of total 5 -FU elimination occurs in the GI tract, while about $23 \%$ is eliminated in the liver (at an infusion rate of 5 -FU of $0.625 \mathrm{mg} \mathrm{kg}^{-1} \mathrm{hr}^{-1}$ ). Assuming that these ratios roughly reflect the relative magnitudes of $V_{\max }^{\mathrm{G}}$ and $V_{\max }^{\mathrm{L}}$ (in humans), then Eq. (A3) becomes:

$$
\varepsilon^{2}=0.39\left(\frac{1}{\lambda}-1\right)+0.61 \varepsilon_{\mathrm{L}}^{2}
$$

where $\lambda$ is the fraction of the total hepatic blood flow $Q$ that passes through the portal vein (see Appendix).

Past estimates of $\varepsilon_{\mathrm{L}}^{2}$ for the liver alone include $\varepsilon_{\mathrm{L}}^{2}=0.12$ for the uptake of taurocholate by isolated perfused rat livers [data of Pries et al. (22), analyzed in ref. 13]; $\varepsilon_{\mathrm{L}}^{2}=0.137$ for uptake of colloidal $\mathrm{CrPO}_{4}$ by rat liver [data of Bauer et al, (14), analyzed in ref. 15]; $\varepsilon_{\mathrm{L}}^{2} \leq 0.182$ for galactose uptake by rat liver [data of Keiding and Chiarantini (17), analyzed in ref. 23], and $\varepsilon_{\mathrm{L}}^{2}=0.196$ for galactose elimination by isolated pig livers [data of Keiding et al. (24), analyzed in ref. 20]. No estimates of $\varepsilon_{\mathrm{L}}^{2}$ for normal human livers exist in the literature. However, it has been shown for galactose elimination that in one cirrhotic patient (data from $\mathrm{K}$. Winkler, private communication), about $36 \%$ of total hepatic blood flow is shunted past the 
functional enzymes (25). Such intrahepatic shunts are a special case of heterogeneity, and an equivalent value for $\varepsilon_{\mathrm{L}}^{2}$ corresponding to a particular shunt can be calculated (19); for a $36 \%$ shunt, $\varepsilon_{\mathrm{L}}^{2}=0.56$. It is, however, unlikely that normal human livers would have such a high degree of intrahepatic shunting.

The magnitude of $K_{\mathrm{m}}^{+}$estimated by means of the distributed sinusoidal perfusion model depends on $\varepsilon^{2}$ according to Eq. (13): the larger $\varepsilon^{2}$, the smaller is the estimated value of $K_{\mathrm{m}}^{+}$. The size of $\varepsilon^{2}$ in turn depends on $\varepsilon_{\mathrm{L}}^{2}$ according to Eq. (17). For the pooled 5-FU uptake data of Wagner et al. (5), $F_{\mathrm{i}}=0.163$ and $V_{\max } / Q=48.0 \mu \mathrm{M}$; for $R=0$, the calculated values of $K_{\mathrm{m}}^{+}$as a function of $\varepsilon_{\mathrm{L}}^{2}$ are shown in Fig. 3 for $\lambda=0.7,0.75$ and 0.8 . Also shown in Fig. 3 are calculated values of $K_{\mathrm{m}}$ and $K_{\mathrm{m}}^{*}$ from the venous equilibration and undistributed sinusoidal perfusion models (horizontal arrows). Values of $\varepsilon_{\mathrm{L}}^{2}$ previously estimated from other data sets are shown as vertical arrows on the abscissa. For $\varepsilon_{\mathrm{L}}^{2}=0.20$ and $\lambda=0.75$ we have from Fig. 3 that $K_{\mathrm{m}}^{+}=23 \mu \mathrm{M}$, and from Eq. (17) that $\varepsilon^{2}=0.125$. From Eq. (15) the remainder term $R$ lies in the range $0.018<R \leq 0.019$, which is less than $10 \%$ of the previous term in the expansion (13), thus justifying its neglect in our calculations of $K_{\mathrm{m}}^{+}$in this case. For larger values of $\varepsilon_{\mathrm{L}}^{2}, R$ becomes relatively larger: for $\varepsilon_{\mathrm{L}}^{2}=0.56, R$ is more than one-third of the previous term in Eq. (13). At such high values of $\varepsilon_{\mathrm{L}}^{2}$, estimates of $K_{\mathrm{m}}^{+}$given by Fig. 3 can be used only as a rough guide. Values of $K_{\mathrm{m}}^{+}$calculated according to Eq. (13) with $R=0, \varepsilon^{2}=0.125$ and using values of $V_{\max } / Q$ for individual patients, are given in Table II. They have a mean value of $20.3 \pm 2.8$ (SEM) $\mu \mathrm{M}$, which is within $13 \%$ of the upper bound to $K_{\mathrm{m}}$ given by the undistributed sinusoidal perfusion model.

The foregoing numerical estimates of kinetic parameters show that the several models differ substantially in their interpretations of the data, so that an ultimate decision between the models is desirable. It is also apparent that such a decision cannot be reached on the basis of clinical data alone: Modeling theory is at present ahead of experiment in the sense that clinical data do not determine all the model parameters unambiguously. We do not view this as a deficiency of the models, because one of the roles of theory is to motivate future experimental designs and methods of measurement.

Whereas validation of models is best based on studies of groups of subjects, the ultimate use of the models pertains to individual patients, as attempted here. Estimates of the kinetic parameters $V_{\max }$ and $K_{\mathrm{m}}$ have evident clinical importance for individual patients, especially for the choice of dosage of toxic drugs such as 5-FU. Whereas a steady infusion rate $I<V_{\max }$ results in a steady, controlled concentration in the body, an increase of $I$ to a value exceeding $V_{\max }$ leads to concentrations tending to arbitrarily large values. While an estimate of $V_{\max }$ is thus especially useful for choices 


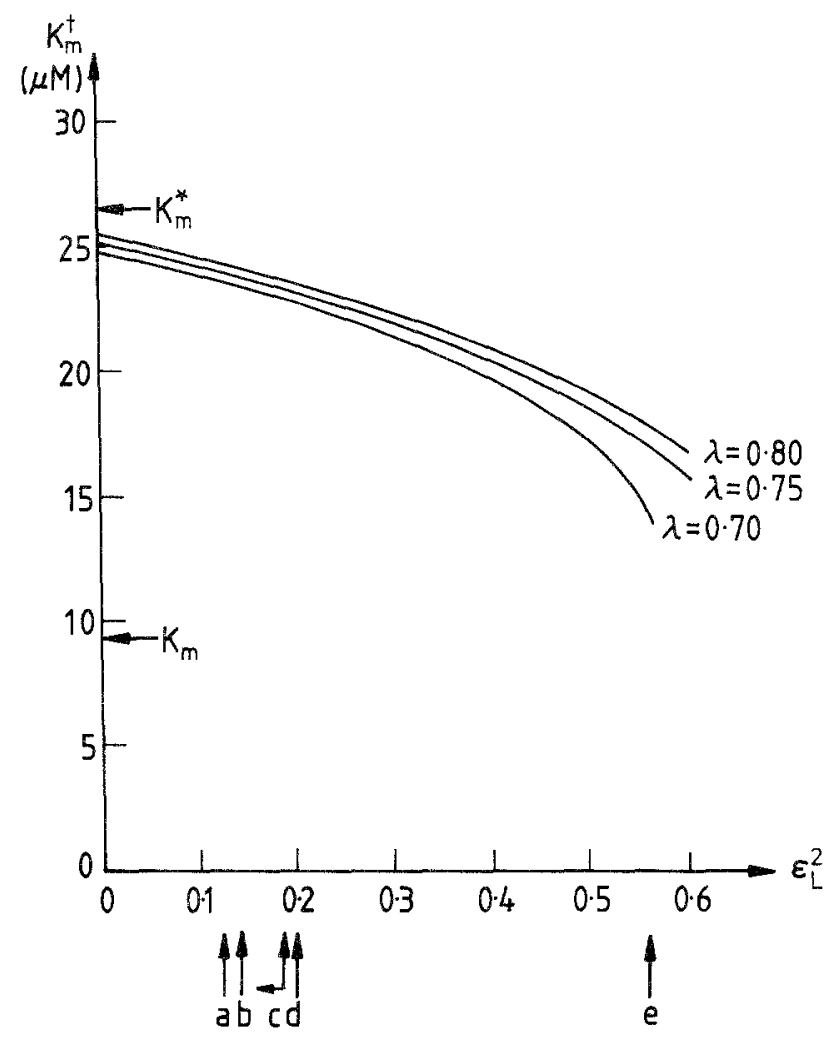

Fig. 3. Variation of $K_{m}^{+}$with the degree of heterogeneity $\varepsilon_{L}^{2}$ of the liver, from the pooled data of Wagner et al. (5) and based on the distributed sinusoidal perfusion model [Eq. (13)], with $V_{\max } / Q=$ $48.0 \mu \mathrm{M}, F_{\mathrm{i}}=0.163$ and $R=0$. Curves are drawn for three values of the fraction $(\lambda)$ of the total hepatic blood flow passing through the portal vein. Total splanchnic heterogeneity $\varepsilon$ is given by $\mathrm{Eq}$. (17). Note that for $\varepsilon_{\mathrm{L}}^{2}=0, K_{\mathrm{m}}^{+}$is still slightly less than $K_{\mathrm{m}}^{*}$ estimated from the undistributed model (upper horizontal arrow) due to heterogeneity associated with the GI tract-hepatic artery system. The lower horizontal arrow shows $K_{\mathrm{m}}$ estimated by the venous equilibration model. Vertical arrows on the abscissa show previously estimated values of $\varepsilon_{\mathrm{L}}^{2}$ : (a) taurocholate elimination by isolated rat livers $(13,22)$; (b) colloidal $\mathrm{CrPO}_{4}$ elimination by $\mathrm{RE}$ system of rat liver $(14,15)$; (c) and (d) galactose elimination by isolated pig livers $(17,20,23,24)$; (e) galactose elimination by cirrhotic human liver (25). 
of steady infusions, an estimate of $K_{\mathrm{m}}$ for each individual patient is useful for controlling the delivery of drugs in bolus inputs. As long as the substrate concentration in the bolus is well below $K_{\mathrm{m}}$ (first-order kinetics), the hepatic extraction fraction is independent of concentration and hence of dose delivery. When the bolus concentration approaches and exceeds $K_{\mathrm{m}}$, the extraction fraction falls as concentration is increased: in the limit $V \rightarrow V_{\max }$, the hepatic extraction fraction tends to zero, and bioavailability $C_{\mathrm{HV}} / C_{\mathrm{HA}}$ tends to unity. As the onset of these nonlinear phenomena is controlled by the value of $K_{\mathrm{m}}$, its estimate (together with that of $V_{\max }$ ) for each individual patient is a prerequisite of rational dosing. Furthermore, changes in the hepatic extraction fraction with changes in hepatic blood flow (in exercise, cardiac failure, after meals, etc.) and with pathological changes in liver heterogeneity (as in intrahepatic shunting in cirrhosis) are of clinical interest. These considerations illustrate the clinical importance of the choices and uses of the models of hepatic elimination.

\section{APPENDIX: ELIMINATION BY LIVER PLUS GI TRACT}

We describe a composite model for steady-state Michaelis-Menten elimination of a substrate by both the liver and the GI tract, as shown schematically in Fig. 4. Blood with substrate at a concentration $C_{\mathrm{HA}}$ enters both the hepatic artery and the GI tract at flow rates $(1-\lambda) F$ and $\lambda F$ respectively, where $F$ is the rate of total hepatic blood flow and $\lambda$ is the fraction of $F$ that is contributed by the portal circulation (Figs. 4 and 1).

Substrate is eliminated from the blood during passage through the GI tract, which outputs into the portal vein. We adopt the common assumption of complete prehepatic mixing of portal and hepatic arterial blood. As a

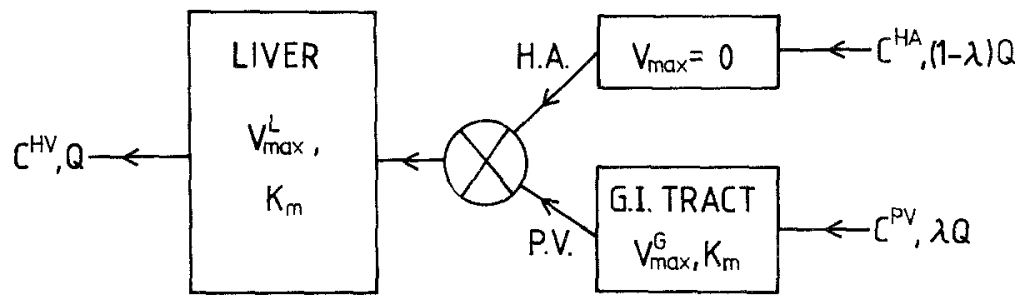

Fig. 4. Schematic diagram of composite model of splanchnic region described in the Appendix, consisting of three suborgans. Arterial blood, concentration $C_{\mathrm{HA}}$, enters the hepatic artery and the portal circulation passing through the GI tract, with flows of $(1-\lambda) Q$ and $\lambda Q$ respectively, where $Q$ is total hepatic blood flow. Substrate is eliminated in the GI tract but not in the hepatic artery; blood from these two parallel suborgans is then mixed before entering the liver, where substrate is further eliminated before being output into the hepatic vein at a concentration $C_{\mathrm{HV}}$. 
result of hepatic elimination, the observed hepatic venous concentration is $C_{\mathrm{HV}}$.

Let the maximum elimination capacity of the liver be given by $V_{\max }^{\mathrm{L}}$, and that of the GI tract by $V_{\max }^{\mathrm{G}}$. Let us assume that the elimination process is such that the Michaelis constant $K_{\mathrm{m}}$ is the same in both liver and GI tract. This assumption is consistent with the finding of similar metabolites of 5-FU in the epithelium of the GI tract as in the liver following intravenous injections of 5-FU in mice (2). The splanchnic region as a whole can then be represented schematically as in Fig. 4, consisting of three suborgans. Of these, the GI tract and the hepatic artery are functionally in parallel (the hepatic artery acts essentially as a shunt). In series with these is the liver itself which receives as its input the mixed output from the GI tract and the hepatic artery. Let us assume, for simplicity, and in the absence of any data concerning its functional heterogeneity, that the GI tract eliminates substrate according to the undistributed sinusoidal perfusion model. Then the composite organ, representing the GI tract and hepatic artery in parallel, can be characterized by the parameter $\varepsilon_{\mathrm{G}}$ which can be calculated from Eq. (14). Letting $Q^{(1)}=\lambda Q, V_{\max }^{(1)}=V_{\max }^{\mathrm{G}}$ for the GI tract, and $Q^{(2)}=(1-\lambda) Q$, $V_{\max }^{(2)}=0$ for the hepatic artery shunt gives, from Eq. (14)

$$
\varepsilon_{\mathrm{G}}^{2}=\frac{1}{\lambda}-1
$$

Since we have neglected any contributions to heterogeneity from the GI tract alone, Eq. (A1) represents a lower bound to the true $\varepsilon_{\mathrm{G}}^{2}$.

The splanchnic region is now reduced to two suborgans in series: the GI tract/hepatic artery suborgan, with $\varepsilon_{\mathrm{G}}^{2}$ given by Eq. (A1), and the liver itself, with heterogeneity characterized by $\varepsilon_{\mathbf{L}}^{2}$. These two suborgans are connected by an intraorgan mixing site. The method of calculation of the composite value for $\varepsilon^{2}$ for a number of distributed suborgans in series, each separated by a site of mixing, has been described previously for first-order uptake of substrate (13), and is given by

$$
\varepsilon^{2}=\sum_{s} \varepsilon_{(s)}^{2}\left[\frac{V_{\max }^{(s)}}{V_{\max }}\right]^{2}
$$

where $s$ denotes elements in series, and where $V_{\max }$ is the total maximum elimination capacity. In our present example, $\varepsilon_{(1)}^{2}=\varepsilon_{\mathrm{G}}^{2}, V_{\max }^{(1)}=V_{\max }^{\mathrm{G}}$, and $\varepsilon_{(2)}^{2}=\varepsilon_{\mathrm{L}}^{2}, V_{\max }^{(2)}=V_{\max }^{\mathrm{L}}$, so that Eq. (A2) gives for the splanchnic region as a whole

$$
\varepsilon^{2}=\left(\frac{1}{\lambda}-1\right)\left[\frac{V_{\max }^{\mathrm{G}}}{V_{\max }}\right]^{2}+\varepsilon_{\mathrm{L}}^{2}\left[\frac{V_{\max }^{\mathrm{L}}}{V_{\max }}\right]^{2}
$$


where $V_{\max }=V_{\max }^{\mathrm{G}}+V_{\max }^{\mathrm{L}}$. Equation (17) is an application of this result. Thus, in the first-order limit, the composite model of the splanchnic region shown in Fig. 4 is described in terms of a single distributed sinusoidal perfusion model with a maximum elimination rate $V_{\max }=V_{\max }^{\mathrm{G}}+V_{\max }^{\mathrm{L}}$, and the value for $\varepsilon^{2}$ given by Eq. (A3).

\section{REFERENCES}

1. J. L. Speyer, P. H. Sugarbaker, J. M. Collins, R. L. Dedrick, R. W. Klecker, Jr., and C. E. Myers. Portal levels and hepatic clearance of 5-fluorouracil after intraperitoneal administration in humans. Cancer Res. 41:1916-1922 (1981).

2. M. Chadwick and J. Rogers. The physiological disposition of 5-fluorouracil in mice bearing solid L1210 lymphocytic leukemia. Cancer Res. 32:1045-1056 (1972).

3. B. G. Gustavsson, A. Brandberg, C. G. Regardh, and O. E. Almersjo. Regional and systemic serum concentration of 5-fluorouracil after portal and intravenous infusion: an experimental study in dogs. J. Pharmacokin. Biopharm. 7:665-673 (1979).

4. Y.-H. Kao, C.-Y. Yang, R. R. L. Chen, and J.-D. Huang. Pulmonary elimination of 5-fluorouracil in anesthetized rats. J. Pharm. Sci. 74:1095-1096 (1985).

5. J. G. Wagner, J. W. Gyves, P. L. Stetson, S. C. Walker-Andrews, I. S. Wollner, M. K. Cochran, and W. P. Ensminger. Steady-state nonlinear pharmacokinetics of 5-fluorouracil during hepatic arterial and intravenous infusions in cancer patients. Cancer Res, 46:14991506 (1986).

6. L. Bass, S. Keiding, K. Winkler, and N. Tygstrup. Enzymatic elimination of substrates flowing through the intact liver. $J$. Theor. Biol. 61:393-409 (1976).

7. J. R. Gillette. Factors affecting drug metabolism. Ann. N.Y. Acad. Sci. 179:43-66 (1971).

8. M. Rowland, L. Z. Benet, and G. G. Graham. Clearance concepts in pharmacokinetics. J. Pharmacokin. Biopharm. 20:123-136 (1973).

9. R. A. Branch, A. S. Nies, and D. G. Shand. The disposition of propranolol VIII. General implications of the effects of liver blood flow on elimination from the perfused rat liver. Drug Metab. Dispos. 1:687-690 (1973).

10. L. Bass, P. J. Robinson, and A. J. Bracken. Hepatic elimination of flowing substrates: The distributed model. $J$. Theor. Biol. 72:161-184 (1978).

11. M. S. Roberts and M. Rowland. Hepatic elimination-dispersion model. J. Pharm. Sci. 74:585-587 (1985).

12. M. S. Roberts and M. Rowland. A dispersion model of hepatic elimination. J. Pharmacokin. Biopharm. 14:227-308 (1986).

13. L. Bass, M. S. Roberts, and P. J. Robinson. On the relation between extended forms of the sinusoidal perfusion and of the convection-dispersion models of hepatic elimination. $J$. Theor. Biol. 126:457-482 (1987).

14. R. W. Brauer, G. F. Leong, R. F. McElroy, and R. J. Holloway. Circulatory pathways in the rat liver as revealed by $\mathrm{P}^{32}$ chromic phosphate colloid uptake in the isolated perfused liver preparation. Am. J. Physiol. 184:593-598 (1956).

15. L. Bass. Flow-dependence of first-order uptake of substances by heterogeneous perfused organs. J. Theor. Biol. 86:365-376 (1980).

16. K. S. Pang and M. Rowland. Hepatic clearance of drugs. II. Experimental evidences for acceptance of the "well stirred" model over the "parallel tube" model using lidocaine in the perfused rat liver in situ preparation. $J$, Pharmacokin. Biopharm. 5:655-680 (1977).

17. S. Keiding and E. Chiarantini. Effect of sinusoidal perfusion on galactose elimination kinetics in perfused rat liver. J. Pharmacol. Exp. Ther. 205:465-470 (1978).

18. S. Keiding and E. Steiness. Flow dependence of propranolol elimination in perfused rat liver. J. Pharmacol. Exp. Ther. 230:474-477 (1984).

19. L. Bass. Saturation kinetics in hepatic drug removal: a statistical approach to functional heterogeneity. Am. J. Physiol. 244:G583-G589 (1983). 
20. L. Bass and P. J. Robinson. Effects of capillary heterogeneity on rates of steady uptake of substances by the intact liver. Microvasc. Res. 22:43-57 (1981).

21. F. Mentre, J. L. Steimer, J. P. Sommadossi, R. B. Diasio, and J. P. Cano. A mathematical model of the kinetics of 5 -fluorouracil and its catabolites in freshly isolated rat hepatocytes. Biochem. Pharmacol. 33:2727-2732 (1984).

22. J. M. Pries, A. B. Staples, and R. F. Hanson. Effect of hepatic blood flow on taurocholate extraction by the isolated perfused rat liver. $J$. Lab. Clin. Med. 97:412-417 (1981).

23. L. Bass and P. J. Robinson. How small is the functional variability of liver sinusoids? J. Theor. Biol. 81:761-769 (1979).

24. S. Keiding, S. Johansen, K. Winkler, K. Tonnesen, and N. Tygstrup. Michaelis-Menten kinetics of galactose elimination by the isolated pig liver. Am. J. Physiol. 230:1302-1313 (1976).

25. P. J. Robinson. Aspects of mathematical liver kinetics: the steady-state statistical mechanics of hepatic elimination. Ph.D. thesis, University of Queensland. (University Microfilms International, Ann Arbor, Michigan, 1979) 